\title{
Aplicação de um instrumento para detecção precoce e previsibilidade de agravos na população idosa
}

\author{
Application of an instrument for detecting \\ and predicting diseases in the elderly people
}

Leticya dos Santos Almeida N egri 1

Gustavo Favarato Ruy 1

João Bosco Collodetti 1

Luiz Felipe Pinto 2,3

Daniel Ricardo Soranz 3,4

1 Secretaria Municipal de Saúde de João Neiva. Rua Sete de Setembro 177, Centro, 29680-000, João Neiva ES.

lenegri01@uol.com.br.

2 Departamento de

Ciências Sociais, Escola

Nacional de Saúde Pública,

Fiocruz. Rio de Janeiro.

3 Fundação Educacional

Serra dos Órgãos, Centro de Ciências Biomédicas.

Teresópolis, Rio de Janeiro.

4 Centro de Saúde Escola

Germano Sinval Faria,

Escola Nacional de Saúde

Pública, Fiocruz.

\begin{abstract}
The increase in the elderly population demands major changes in health care models for detecting and predicting diseases which seize the elderly whose health care demands a higher concern considering that most of them, $80 \%$ at least, have one chronic disease. In quest for an adequate health care for the elderly, this study - marked by an exploring and cross-sectional character - de cided to apply a short Veras questionnaire for an intentional sample of 103 elderly people which used the services on a typical week in two medical facilities from the County of João N eiva (ES). One is in the rural area (a health family team from the District of Acioli) and another onein the urban area (which cares for spontaneous and scheduled patients). This protocol allows for a quick evaluation of the physical conditions of the patient in different groups at risk and frailty levels which they are subjected to. The high risk for the "frailty groups" in João N eiva is superior to that of the rural area $(54,6 \%)$ when compared to the urban area $(42,4 \%)$. The observed factors of risk which turned out to be more important for predicting diseases were gender, age, self- perception of health and hospital admissions in the last twelvemonths previous to theinterview - tools used to classify the elderly in different frailty groups. It's suggested to test the validity of the method in a prospective study, with a higher sample.

Key words Elderly population, Predicting diseases, Public health, Frailty, Family health
\end{abstract}

Resumo 0 aumento da população idosa exige mudanças nos model os de assistência e previsão das doenças que acometem os idosos. Trata-se de um grupo etário cujos cuidados com a saúde devem ser maiores, uma vez que $80 \%$ de seus integrantes apresentam pelo menos uma enfermidade crônica. Em busca de uma assistência adequada à saúde do idoso, este estudo de natureza exploratória e corte transversal optou por aplicar um questionário a uma amostra intencional de 103 idosos que utilizaram os serviços numa semana típica de duas unidades de saúde do município de João N eiva (ES). U ma das unidades está na área rural, que possui uma equipe de saúde da família, e a outra na área urbana, que desenvolve atendimento com demanda espontânea eagendada. Este protocolo permite avaliar o quadro físico do paciente em diferentes grupos de riscose grau de fragilização a que estão sujeitos. 0 alto risco de fragilização da população idosa de João $N$ eiva é superior na área rural $(54,6 \%)$ quando comparado à área urbana $(42,4 \%)$. Os fatores de risco observados mais importantes para a previsibilidade de agravos foram gênero, faixa etária, autopercepção de saúde, e internação nos últimos 12 meses anteriores à entrevista. Sugere se testar a validade do método em um estudo prospectivo, com uma amostra de tamanho maior.

Palavras-chave Idosos, Previsibilidade de agravos, Saúde coletiva, Fragilização, Saúde da família 


\section{Introdução}

No Brasil, em 1900, a expectativa de vida ao nascer era 33,7 anos. Pouco tempo depois, nos anos 40, passou para 39 anos. Nos anos 50, atingiu 43,2 anos, ena década de 1960, 55,9 anos. De 1960 para 1980, essa expectativa ampliou-se para 63,4 anos, isto é, foram acrescidos quase dez anos, em três décadas, segundo revelam os dados do Anuário Estatístico do Brasil de 1982 (IBGE, 1982). De 1980 para 2000, o aumento estimado foi de cinco anos, ocasião em que cada brasileiro, ao nascer, esperava viver 68,5 anos (IBGE, 2000a).

As projeções para o período de 2000 a 2025 permitem estimar uma expectativa média de vida do brasileiro próxima dos 80 anos, atingindo, enfim, os níveis de países desenvolvidos observados em 2000. Nos dias de hoje, o relógio biológico do ser humano atinge 90-95 anos (Veras, 2001; Veras et al., 2002). Segundo estes autores, al gumas estimativas prevêem que nas próximas décadas a tendência desse indicador éaumentar, al cançando 120-130 anos.

Paralelamente a este aumento na expectativa de vida a partir da década de 1960, ocorreu um declínio acentuado da fecundidade, levando a um aumento importante da proporção de idosos na população brasileira, sendo estes definidos como o total de pessoas com 60 anos e mais (RIPSA, 2002).

Nos países desenvolvidos o processo de envelhecimento ocorreu de forma lenta e acompanhou a elevação da qualidade de vida das populações urbanas e rurais. No Brasil, o envelhecimento é um fenômeno predominantemente urbano ( $82 \%$ dos idosos estão morando em cidades) e o processo de transição demográfica caracteriza-se pela rapidez com que 0 aumento absoluto e relativo das populações adultas e idosas vem modificando a pirâmide populacional.

Carvalho \& Garcia (2003) alertam para o fato de que, ao contrário do imaginário comum, o processo de envelhecimento populacional, tal como observado até hoje, é resultado do declínio da fecundidade, e não da mortalidade. No Brasil, o declínio da fecundidade ocorreu em um ritmo maior e origina-se de uma população mais jovem do que aquela dos países desenvolvidos. Estudos têm mostrado o aumento da população idosa em todo o mundo e também no Brasil (Ramos, 1993). Estas pessoas deveriam ter a assistência de profissionais preparados especialmente para atendê-las, uma vez que a abordagem médica tradicional do adulto hospi- talizado centrada na queixa principal, bem como 0 hábito de o profissional médico tentar explicar todas as queixas e os sinais por um único diagnóstico, é procedimento adequado ao adulto jovem, mas não se aplica ao idoso. Um alerta aos gestores do Sistema Ú nico de Saúde foi revelado através de um estudo nacional inédito desenvolvido por Barros Silva et al. (2001) apontando que no SUS apenas $60 \%$ dos municípios brasileiros possuem atividades voltadas à saúde do idoso.

Outros inquéritos populacionais demonstram que a maioria dos idosos (80\%) apresenta pelo menos uma doença crônica, e uma significativa parcela, $33 \%$, três ou mais agravos (IBGE, 2000a).

Para Ramos (1993), a falta de conhecimentos geriátricos tem contribuído decisivamente para as dificuldades na abordagem médica do paciente idoso. Além disso, Reuben et al. (2003) ressaltam que alguns métodos de avaliação da qualidade da atenção ao idoso têm sido imperfeitos ao longo das últimas décadas.

Do ponto de vista da saúde pública, a capacidade funcional (capacidade de manter as habilidades físicas ementais para uma vida independente eautônoma) surge como um novo conceito, mais adequado, para instrumentalizar e ope racionalizar a atenção à saúde do idoso. N esse sentido, ações preventivas, assistenciais e de reabilitação para melhoria da capacidade funcional ou, no mínimo, a sua manutenção são fundamentais para a qualidade de vida dos idosos.

No contexto da recente edição da Política Nacional de Saúde do Idoso, e do vertiginoso processo de envelhecimento que ocorre no Brasil, espera-se que, em todos estados e municípios, consiga-se avançar no processo de consolidação do SUS, regulamentado pela Constituição Federal de 1988, e pelas Leis Complementares, obtendo-se de fato a universalização e a integralidade da atenção à saúde do idoso. Promover o envelhecimento saudável, ou seja, envel hecer com preservação da capacidade funcional, autonomia e qualidade de vida, por maior tempo possível, éa principal meta da Política do Idoso (Brasil, 2003).

0 grande desafio para todos nesse novo milênio éa construção de uma consciência coletiva de forma que seja encarada com justiça social e garantia plena de direitos independente da faixa etária. Ao assumir, em 1994, a Estraté gia Saúde da Família, como uma proposta de reorganização da atenção básica e de mudança de modelo assistencial, acima de tudo, resoluti- 
va e de boa qualidade à população, as equipes vêm identificando fatores de risco, nos quais à população está exposta, e neles, atuando de forma apropriada. A abordagem do idoso na Estratégia de Saúde da Família consiste em mantê-lo na comunidade, com o apoio social junto de sua família, da forma mais digna e confortável possível. Seu deslocamento para um serviço de longa permanência, seja um hospital, asilo, casa de repouso ou similar, é uma al ternativa que só pode ser considerada quando faltarem todos os esforços possíveis para a promoção, manutenção e recuperação de sua capacidade funcional. Tanto a internação dos idosos em serviços de longa permanência, como a internação hospitalar por curto prazo de tempo, representa um modelo excludente e que causa graves prejuízos a sua capacidade funcional e autonomia (Silvestre $\&$ Costa Neto, 2003).

No contexto da Saúde da Família, destacase o trabalho dos profissionais de saúde voltado para a assistência integral e contínua de todos os membros da família, vinculados à Unidade Básica de Saúde (UBS), em cada fase de seu ciclo de vida, sem perder de vista o seu contexto familiar e social. A equipe de saúde da família deverá estar atenta à mudança do perfil populacional em sua área de abrangência, a partir do envelhecimento da população, evitando sempre que possível a apartação do idoso do convívio familiar e social. N esse sentido, uma publicação recente do M inistério da Saúde, referencia que (...) a equipe de saúde da família precisa estar atenta à pessoa idosa, na constante atenção ao seu bem-estar, à sua rotina funcional ea sua inserção familiar e social, jamais a deixando à margem de seu contexto, mantendo-a mais independente possível no desempenho de suas atividades rotineira (M inistério da Saúde, 2002).

A utilização de um instrumento que possibilite a determinação do "grau de fragilização" fornece a possibilidade de estabelecer limites entre o que se pode considerar envelhecimento normal, com suas limitações fisiológicas gradativas, e as características patológicas que podem instituir-se durante esse processo. 0 objetivo desse instrumento, além da identificação precoce de agravos, é permitir o desenvolvimento de ações de caráter individual e coletivo, visando à prevenção específica, o diagnóstico precoce e o tratamento adequado dos principais problemas de saúde desse segmento populacional. Vale ressal tar que o Brasil é um país que envelhece de maneira rápida e o envel hecimento populacional aumentará em conseqüência dos avanços nos conhecimentos da engenharia genética e da biotecnologia, alterando, em um futuro próximo, não apenas indicadores demográficos como a expectativa de vida, mas principalmente 0 próprio limite do tempo de vida. Em menos de 40 anos, o Brasil passou de um perfil de mortalidade típico de uma população jovem para um quadro caracterizado por enfermidades complexas e onerosas, próprias das faixas etárias mais avançadas. Esse fato exige um profissional de saúde mais capacitado e que tenha domínio de instrumentos, como o que está sendo proposto neste trabalho. Com este instrumento, é possível fazer a detecção precoce e previsibilidade de agravos na população idosa, e identificar o grau de fragilização dos pacientes.

Segundo o Censo Demográfico de 2000 (IBGE, 2000a), o Estado do Espírito Santo tem 2,6 milhões de habitantes, dos quais $6,7 \%$ possuem 60 anos ou mais. No município de João Neiva, onde o estudo foi desenvolvido, de um total de 15.863 habitantes, 1.709 pessoas possuíam 60 anos ou mais, o que representava $10,8 \%$ da população total, ou seja, trata-se de um município com cerca de $50 \%$ a mais de população nessa faixa etária que a média geral do estado. A elevada freqüência de idosos em João N eiva sugere que os gestores da área de saúde passem a considerar a necessidade de mudança nos modelos assistenciais, tendo em vista a significativa ampliação dos custos decorrentes da atenção à saúde desse segmento populacional. Este artigo tem como objetivo central comparar o perfil de risco dos idosos que freqüentam duas unidades de saúde: uma localizada em área urbana, com demanda espontânea e agendada, e outra em área rural, que possui uma equipe de saúde da família, no município de João Neiva (ES). Concomitantemente, será descrito 0 perfil sociodemográfico, epidemiológico e aspectos do financiamento em saúde.

\section{Material e métodos}

0 estudo, de natureza descritiva e exploratória, apresenta uma abordagem quantitativa, baseada principalmente em pesquisa de campo (Tobar \& Yalour, 2001), além de ter considerado um levantamento de alguns bancos de dados secundários (IBGE, SIM, SINASC, SIAB, SIA, SIH eSIOPS). Foi selecionada uma amostra intencional de tamanho $n=103$, a partir de idosos que freqüentaram uma semana típica de um mês ( outubro de 2003), em duas unidades de 
saúde de João Neiva: uma localizada na área urbana (Centro - João N eiva) e outra na área rural (unidade de saúde da família, Distrito de Acioli). A unidade situada na área urbana atende a demanda espontânea e agendada. Por isso, quando comparado ao estudo realizado por Veras (2003), nosso levantamento de dados reve lou uma proporção maior de idosos com risco de fragilização médio/alto e alto. Esse fato justifica-se pelo elevado contingente de pessoas que utilizaram os serviços no período de referência da pesquisa como segunda consulta. 0 estudo se baseia, portanto, mais na vertente de "previsibilidade de agravos" do que detecção precoce ("triagem rápida") descrita por este autor. Além disso, a estratégia de saúde da família no município de João Neiva possui uma cobertura de sua população-residente próxima de $100 \%$, 0 que também a diferencia em relação ao inqué rito desenvolvido por Veras (2003).

\section{Atendimento ao idoso nas unidades de saúde}

Os idosos foram abordados no período de 13 a 17 de outubro, quando chegavam às duas unidades, durante seu horário de funcionamento: das 7 às 11 h, e entre 12 e 16h, sendo informados do desenvolvimento e objetivos do estudo e fornecendo seu consentimento livre e esclarecido através de formulário próprio elaborado pela equipe técnica. 0 entrevistado ficava então ciente de que sua identidade seria mantida em sigilo, e que poderia desistir da participação, a qualquer momento da entrevista; e ainda que as informações seriam utilizadas somente para a pesquisa em questão, assim como sua assistência no serviço de saúde não sofreria qualquer prejuízo. Os questionários foram aplicados em pessoas com 60 anos ou mais, que procuravam as unidades de saúde nesse período, que representa uma semana típica de atendimento, sendo 71 na unidade de saúde localizada na área urbana e 32 na unidade de saúde da família, situada na área rural. Destaca-se que, dos 71 entrevistados na área urbana, 12 residiam no campo. Como o estudo previa a avaliação do risco em relação ao local de moradia, consideramos, então, que os 12 entrevistados na unidade localizada na área urbana fossem alocados para a área rural, local de residência destes idosos. Com isso, o total de pessoas avaliadas na área urbana foi de 59 pessoas, ena área rural, 44, perfazendo um total de 103 entrevistas, o que, em termos proporcionais, corresponde a cerca de $6 \%$ do total de idosos residentes no município (IBGE, 2000a).

\section{Fragilidade e probabilidade de risco}

A saúde não éuniforme em todos os indivíduos, assim como as doenças. Alguns indivíduos podem atingir idades avançadas em excelente estado de saúde. Entretanto, deve-se ter em mente que esta é a exceção e não a regra. Envelhecer sem nenhuma doença crônica, também. No entanto, a presença de uma doença crônica não implica que o idoso não possa gerir sua própria vida e encaminhar o seu dia-a-dia de forma totalmente independente. Estudos com base populacional têm demonstrado que no Brasil cerca de $80 \%$ dos idosos apresentam, pelo menos, uma enfermidade crônica e, cerca de $33 \%$, pelo menos três enfermidades. No entanto, ao serem perguntados sobre sua saúde, a metade considerou regular; $32 \%$ classificaram-na como boa ou ótima; e somente $22,6 \%$ referenciaram seu estado de saúde como ruim/muito ruim (IBGE, 2000b). Essa demonstração conduz ao quadro de fragilidade de risco do idoso, em que $6 \%$ dos idosos apresentam formas graves de dependência funcional (risco alto); 7 a 10\%, formas moderadas (risco médio); e 25 a 30\% formas leves (risco baixo). Somente 50 a $60 \%$ dos idosos se riam completamente independentes.

A população idosa registra uma fragilização e probabilidade de agravos maiores e, em conseqüência, é a que proporcionalmente consome mais serviços de saúde. Mas, como o sistema de atenção ao idoso é, em muitas localidades do País, precário e desorganizado, não fornece condições para que os recursos sejam utilizados melhor e mais adequadamente. 0 clínico geral que faz atendimento de geriatria vai precisar de instrumentos que 0 ajudem a perceber uma identificação mais correta possível dos distúrbios, a fim de que seja capaz de saber como tratar, e/ou quando encaminhar, o paciente que tem em suas mãos, para um profissional com treinamento espećfico em envelhecimento humano (Veras, 2003). Isto, entretanto, não quer dizer que o volume de recursos (despesas) empregados na tentativa de cuidar dos idosos seja maior. 0 que vale ressaltar é que seria possível gozar de melhor saúde e gastar menos em hospitalização e recursos de complexidade de tecnologia. Com certeza, haveria menores riscos, se as doenças fossem identificadas precocemente e de forma preventiva. 
No Espírito Santo, segundo dados da Pesquisa Nacional por Amostra de Domicílios (PNAD/ 1998), cerca de $30 \%$ dos idosos possuem planos de saúde (IBGE, 2000b). 0 acesso ao atendimento odontológico também tem um grau de importância muito elevado, uma vez que, assim como outra doença física, uma doença bucal pode comprometer a saúde do idoso e identificar certo grau de fragilização. A falta de saúde bucal pode facilmente levar a um quadro de doença cardíaca, como a "endocardite bacteriana", que não deixa de ser uma doença de alto risco.

0 "grau de fragilização" é definido, neste estudo, como sendo a probabilidade de internações repetidas, tal como calculado por Veras (2003) através de um modelo de regressão logística, podendo ser estratificado de diversas maneiras, de acordo com o tipo de avaliação de sejada. Por exemplo, Boult et al. (1993) ao analisarem o protocolo proposto para admissão hospitalar de idosos, definiram apenas dois estratos: 1) um relativo ao risco de menor de 0,5; 2) segundo, com risco igual ou superior a 0,5. Veras (2003), ao discutir uma proposta de fluxo de acesso e definição de prioridades em uma rede ambulatorial, considerou quatro estratos: 1 ) menor de 0,$3 ; 2$ ) entre 0,3 e 0,499; 3) entre 0,4 e 0,499 ; 4) 0,5 ou superior.

Esse estudo, por ter incluído não apenas a primeira consulta de triagem de idosos, mas também, e principalmente, pessoas agendadas para uma segunda ou terceira consulta em uma unidade de saúde da família, redefiniu os pontos de corte, estratificando a probabilidade de internações repetidas em quatro grupos distintos, privilegiando riscos maiores (Tabela 1).

A Política Nacional do Idoso (Brasil, 2003) e a RIPSA (2002) definem como idosos os indivíduos de 60 anos ou mais. Dados estatísticos mostram, porém, a necessidade de intervenção entre idosos mais jovens que compõem a maior parte de população brasileira. No protocolo proposto, os idosos de risco zero são cadastrados e convidados a retornar ao serviço dentro de três meses para nova avaliação, já que no momento da avaliação não apresentaram um perfil de risco que justificasse uma ação imediata, a não ser no caso de uma virose, por exemplo, que deverá ter um tratamento imediato.

Os riscos 1, 2 ou 3 (médio, médio-alto e alto) indicam que o fluxo de atendimento a esses idosos deve se iniciar com marcação de consulta ambulatorial com um clínico, sucedida, mais tarde, por uma avaliação funcional, seguindo as
Tabela 1

Estratos de risco de fragilização.

\begin{tabular}{lll}
\hline Nível & $\begin{array}{l}\text { Risco de } \\
\text { fragilização }\end{array}$ & $\begin{array}{l}\text { Estratificação } \\
\text { deriscos }\end{array}$ \\
\hline 0 & $<0,400$ & Baixo \\
1 & $0,400-0,599$ & M édio \\
2 & $0,600-0,799$ & M édio-alto \\
3 & $>0,800$ & Alto \\
\hline
\end{tabular}

Fonte: D efinição dos autores, a partir de revisão da literatura (Boult et al., 1993; Veras, 2003).

etapas do fluxo de cuidados com o idoso. Com 0 auxílio desse instrumento, os idosos que fossem hospitalizados duas ou mais vezes em um período de quatro anos, seriam rapidamente identificados como "pacientes de alto risco" e beneficiados com programas preventivos, desenvolvidos em unidades de avaliação e reabilitação geriátrica.

Segundo dados da Secretaria M unicipal de Saúde (2003), a prestação de serviço de saúde nos postos de atendimento, inclusive em João Neiva, é feita da mesma forma que na maioria dos outros postos. Desenvolvido em etapas ( $\mathrm{Fi}$ gura 1), o serviço de saúde compreende: 1) captação de clientes: os pacientes dirigem-se aos postos de saúde e marcam as consultas. Vale ressaltar que os postos de saúde atendem pessoas de todas as faixas etárias, desde a infância até a velhice; 2) no dia agendado (ou sefor o caso, no mesmo dia) retornam ao posto de saúde e são encaminhados ao médico; 3) o paciente aguarda na sala de espera a sua chamada. Durante esse tempo, 0 atendente avalia a pressão arterial; 4) após o registro da pressão arterial, 0 paciente é encaminhado ao médico; 5) depois de ter examinado o paciente, o médico faz 0 diagnóstico e, conforme o seu estado, encaminha-o em duas direções: a) o paciente deve participar do acompanhamento médico na própria unidade de saúde; $b$ ) havendo registro de gravidade do quadro, o paciente é encaminhado a um especialista.

\section{Instrumento de triagem para detecção precoce e de possibilidade de agravos}

0 instrumento utilizado foi adaptado a partir dos estudos de Boult et al. (1993) eVeras (2003), sendo aplicado por esteúltimo em uma policlínica na capital do Estado do Rio de Janeiro. Es- 


\section{Figura 1}

Organização atual do serviço de saúde de João N eiva (ES) - 2003

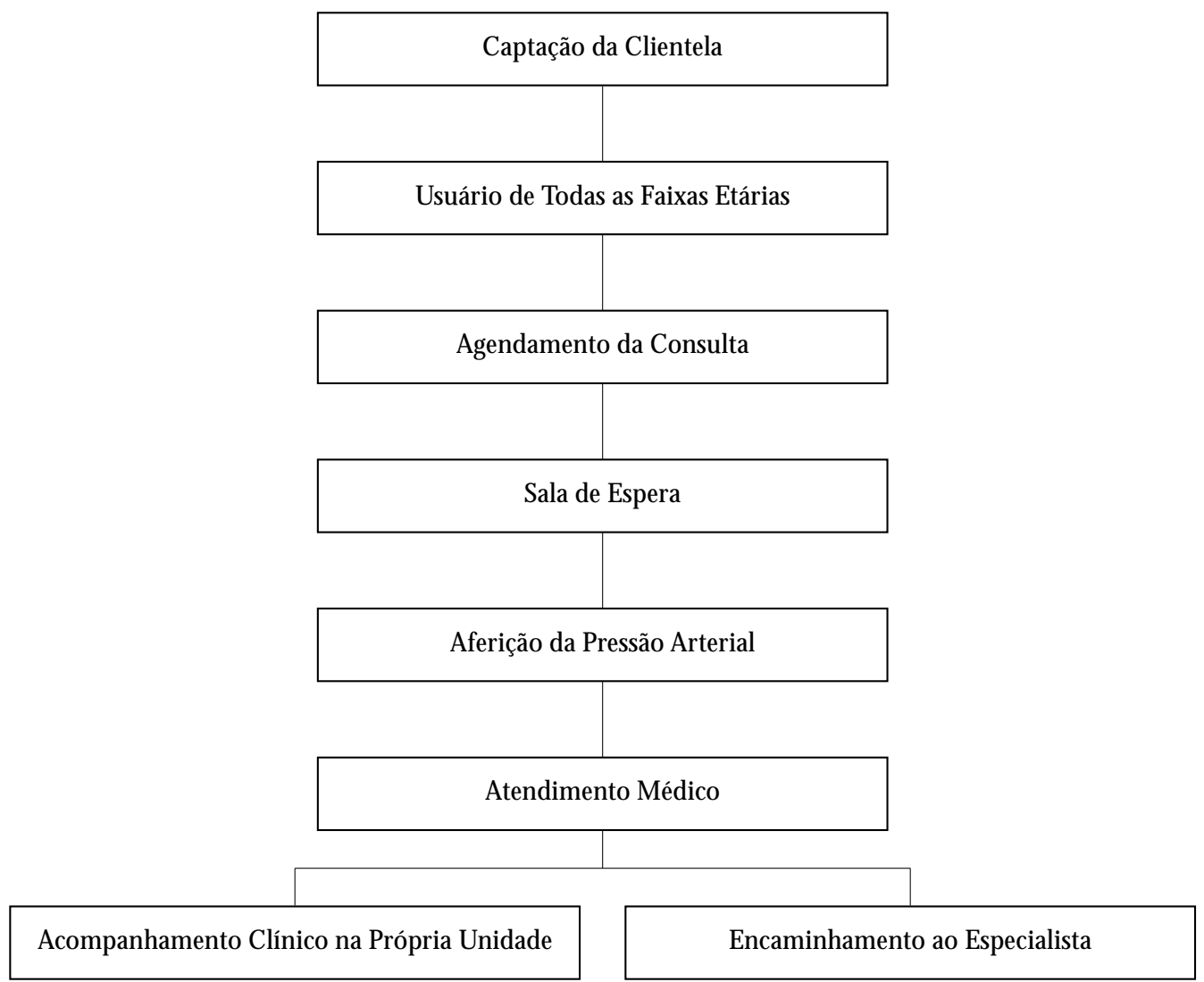

Fonte: Elaboração própria dos autores

te instrumento constitui-se de um breve questionário, com algumas perguntas objetivas, visando à captação e ordenação de idosos, para posterior cálculo de seu grau de fragilização. Em geral, as doenças dos idosos são crônicas e múltiplas, perduram por vários anos e exigem acompanhamento de médico constante e medicação contínua. 0 hábito de 0 médico reunir queixas e sinais do idoso em um único diagnóstico pode ser perigoso. Ao estimar-se a chance de repetidas internações de uma determinada população, Boult et al. (1993) avaliam a presença de um perfil de fragilização, que, além de aumentar a probabilidade de internações repetidas, leva à redução progressiva da capacidade funcional e, conseqüentemente, à maior demanda por serviços de saúde em todos os níveis.

0 objetivo desse instrumento é classificar idosos em diferentes grupos de risco de fragili- zação, possibilitando a hierarquização dos riscos na população e contribuindo para um sistema de saúde mais eficaz e que gere resultados, impedindo queo primeiro atendimento ao idoso ocorra, quando já se encontra em estágio avançado, que fique hospitalizado, fato que não só aumenta os custos como diminui as chances de um prognóstico favorável (M endes, 2001).

Veras (2003) atesta a aplicação desse instrumento, demonstrando alguns dados coletados, nos quais em uma amostra de 360 indivíduos, evidenciou-se que 75,8\% de idosos apresentavam baixo risco de adoecer. Cerca de $11 \%$ da amostra, contudo, apresentou risco de médio a alto de adoe cer, utilizando de forma intensiva os serviços de saúde do pronto atendimento (Veras, 2003). Boult et al. (1993) destacam a vantagem desse simples instrumento, afirmando que com o auxílio desse breve questionário, com apenas 12 pergun- 
tas, os idosos de alto risco, identificados como pacientes hospitalizados duas ou mais vezes em um período de quatro anos, passariam a dispor de um instrumento confiável para indicação de candidatos a atividades de promoção da saúde. Esse questionário contempla as variáveis idade, sexo, renda, disponibilidade de cuidados familiares ("apoio social"), autopercepção de saúde ("morbidade referida"), presença de doença cardíaca, presença de diabetes mellitus, pernoite hospitalar, consultas médicas nos últimos 12 meses, tipo de demanda, acesso médico-odontológico, se possui plano privado de saúde e se ocorreram mais do que duas internações nos últimos quatro anos.

\section{Aspectos sociodemográficos}

O município de João Neiva está localizado no Estado do Espírito Santo e encontra-se em gestão plena do sistema municipal segundo a NOB/96, com uma área geográfica de apenas 281 km², e densidade demográfica de 54,4 hab/km², muito semelhante à concentração populacional observada no município vizinho de lbiraçu. Limitase ao norte com Colatina, ao sul com I biraçu, ao leste com Linhares e Aracruz, e a oeste com Santa Teresa e Colatina. Posiciona-se a 83 quilômetros de distância de Vitória, capital do Espírito Santo. 0 município possui ainda um Conselho Municipal de Saúde composto por 10 membros e respectivos suplentes.

Segundo dados do Censo Demográfico do IBGE (2000a), no ano de 2000, dentre as pessoas responsáveis pelos domicílios particulares permanentes, o número médio de anos de estudo entre os homens era de 5,4 anos e entre as mulheres 4,3 anos. 0 valor do rendimento médio dos chefes de família era de $\mathrm{R} \$ 681$, no caso de homens, e de R\$413, para as mulheres, ou seja, os homens, chefes de família, ganhavam, em média, $65 \%$ a mais que as mulheres. Entre aqueles com 10 anos ou mais, a proporção de analfabetos é pequena, 10,4\% , o que comparado com os demais municípios do Espírito Santo coloca João Neiva no grupo dos 12 municípios com menores taxas de anal fabetismo. Além disso, se considerarmos o analfabetismo funcional entre os chefes de família, ou seja, pessoas que possuem menos de quatro anos de estudo, encontraremos resultado semelhante, com o município ocupando a 15a posi ção geral no Estado, que possui 78 municípios. Um indicador que vem sendo proposto pelo IBGE para medir o nível de pobreza de uma população em seu município natal se refere à proporção de domicílios com saneamento não adequado, cujos responsáveis têm menos de quatro anos de estudo e rendimento mensal de até dois salários mínimos. A análise desse indicador entre os Censos Demográficos de 1991 e 2000 revela o grande avanço social ocorrido em João Neiva, cujos domicílios nessa situação específica passaram de 21,3\% em 1991 para apenas 7,6\%, uma das menores taxas do Estado do Espírito Santo. A análise dos dados de abastecimento de água, instalação sanitária e coleta de lixo pode também ser realizada de forma univariada. Nesse caso, observa-se que houve uma melhoria em todos os indicadores, especialmente o aumento dos domicílios com rede geral de abastecimento de água, que, entre 1991 e 2000, passou de $76,8 \%$ para $85,7 \%$; dos domicílios com rede geral de esgoto ou rede pluvial, de 50,8\% para $74,9 \%$; e o lixo coletado, que teve seus índices melhorados de 64,1\% para 84,1\%. Segundo dados mais recentes do IDH, entre os 78 municípios do Espírito Santo, João N eiva está classificado em 14o lugar; na classificação nacional encontra-se na 1.445a posição. Segundo dados do IBGE (2000a), a taxa de crescimento populacional estimada para o período de 1996 a 2000 foi de $1,8 \%$ ao ano, e a esperança de vida ao nascer, 69 anos.

Como o PSF possui uma cobertura de quase $100 \%$, podemos comparar as contagens realizadas pelo IBGE e pelos agentes comunitários de saúde no PSF. Há uma pequena diferença entre os dados do SIAB e os do IBGE, que estimou, para 2003, uma população-residente de 15.863 pessoas (mais 259 habitantes), sendo 7.930 do sexo masculino e 7.933 do sexo feminino, ou seja, uma razão de sexos - número de homens sobre o número de mulheres - de 99,96. Por outro lado, se analisarmos os dados por faixa etária, constataremos que existe um sobreregistro de pessoas na contagem do IBGE quando comparada ao SIAB nas faixas etárias mais jovens. É de grande importância destacar ainda que há no município uma população de 1.709 pessoas com 60 anos ou mais, sendo 811 do sexo masculino e 899 do sexo feminino, o que re flete a sobrevida das mulheres nas faixas etárias mais avançadas (Tabela 2). Essa diferença entre as duas contagens pode ser explicada, em parte, devido ao fato de ter havido, na metade do ano de 2003, uma redução do número de equipes de saúde da família, passando o município a contar com três unidades. 
Tabela 2

População residente por faixa etária e sexo. João Neiva - 2003.

\begin{tabular}{|c|c|c|c|c|c|c|c|}
\hline \multirow[t]{3}{*}{ Faixa Etária } & \multicolumn{3}{|c|}{ IBGE } & \multicolumn{3}{|c|}{ SIAB } & \multirow{3}{*}{$\begin{array}{c}(\%) \\
\text { (b)/(a) }\end{array}$} \\
\hline & \multicolumn{2}{|c|}{ Sexo } & \multirow{2}{*}{$\begin{array}{c}\text { Total } \\
\text { (a) }\end{array}$} & \multicolumn{2}{|c|}{ Sexo } & \multirow{2}{*}{$\begin{array}{c}\text { Total } \\
\text { (b) }\end{array}$} & \\
\hline & M asculino & Feminino & & Masculino & Feminino & & \\
\hline Menor 1 & 114 & 121 & 235 & 70 & 57 & 127 & 0,54 \\
\hline 1 a 4 & 482 & 466 & 948 & 431 & 421 & 852 & 0,90 \\
\hline 5 a 9 & 732 & 685 & 1.417 & 616 & 616 & 1.232 & 0,87 \\
\hline 10 a 14 & 736 & 764 & 1.500 & 693 & 672 & 1.365 & 0,91 \\
\hline 15 a 19 & 848 & 791 & 1.639 & 732 & 755 & 1.487 & 0,91 \\
\hline 20 a 39 & 2.622 & 2.630 & 5.252 & 2.647 & 2.669 & 5.316 & 1,01 \\
\hline 40 a 49 & 1.037 & 1.010 & 2.047 & 1.033 & 1.079 & 2.112 & 1,03 \\
\hline 50 a 59 & 548 & 568 & 1.116 & 664 & 654 & 1.318 & 1,18 \\
\hline 60 e mais & 811 & 898 & 1.709 & 830 & 965 & 1.795 & 1,05 \\
\hline Total & 7.930 & 7.933 & 15.863 & 7.716 & 7.888 & 15.604 & 0,98 \\
\hline
\end{tabular}

Fonte: IBGE. Estimativas populacionais e SIAB, 2003. Secretaria M unicipal de Saúde de João N eiva/ES.

Além disso, pode-se perceber que a população idosa de João Neiva é proporcionalmente maior que a do Estado do Espírito Santo. Isto sugere que o município é escolhido pela população de idade mais avançada para envelhecer e/ou os jovens migram para as cidades de maior porte em busca de estudo e trabalho, não retornando a sua terra natal.

\section{Perfil da atenção à saúde: capacidade instalada, oferta de serviços, aspectos epidemiológicos e financiamento em saúde}

No contexto da proposta da NOAS/2001, a Se cretaria Estadual de Saúde propôs recentemente no Plano Diretor de Regionalização (SES, 2003) a criação da microrregião de saúde de Linhares, composta pelos municípios de Linhares, Aracruz, Rio Bananal, Sooretama, João Neiva e Ibiraçu, que juntos totalizam uma população estimada, em 2002, de 244.403 habitantes. Os municípios de Linhares e Aracruz são os mais importantes, tanto em extensão territorial, quanto em população e dimensão econômica. Excetuando Aracruz e Rio Bananal, os demais municípios situam-se ao longo da BR-101.

$A$ atenção à saúde pode ser avaliada através da estrutura da rede ambulatorial e hospitalar, além da oferta de serviços e de indicadores epidemiológicos. Em dezembro de 2002, a rede ambulatorial do município era formada por quatorze unidades, sendo quatro postos de saúde $(28,6 \%)$, três unidades de saúde da família $(21,4 \%)$, um centro de saúde $(7,1 \%)$, um am- bulatório de unidade hospitalar especializada $(7,1 \%)$, uma unidade mista $(7,1 \%)$, um consultório $(7,1 \%)$, uma clínica especializada $(7,1 \%)$, um centro/núcleo de atenção psicossocial $(7,1 \%)$ e uma unidade de vigilância sanitária $(7,1 \%)$.

Em relação à quantidade aprovada de procedimentos ambulatoriais, $62,3 \%$ se referem à atenção básica (grupos de 1 a 5 do SIA-SU S), $37,5 \%$ a procedimentos de média complexidade e apenas $0,2 \%$ se refere à al ta complexidade. Entre os procedimentos de atenção básica, realizam-se com maior freqüência ações de enfermagem $(30,2 \%)$, ações básicas de odontologia (14,6\%) e ações médica básicas (11,0\%). Chama a atenção o fato de os procedimentos básicos de odontologia ocuparem a segunda posição, tendência não observada para o Estado do Espírito Santo e também para o Brasil. Essa posição é historicamente ocupada pelas "ações médicas básicas". Já em relação aos procedimentos especializados, sobressaem-se as consultas de fisioterapia (12,3\%), os procedimentos especializados por profissionais médicos ou outros de nível superior (12,0\%), patologia clínica $(8,7 \%)$ e radiodiagnóstico ( $2,9 \%)$. Aqui também registramos uma tendência diferente daquela observada para o Brasil, ou seja, as consultas de fisioterapia destacam-se em primeiro lugar dentre os procedimentos de média complexidade, o que no caso do estudo com a população idosa indica uma boa relação de proximidade entre as necessidades deste segmento etário e a oferta dos referidos serviços em João Neiva. A oferta de leitos em João N eiva é de 44 unidades, o correspondente à capacidade instalada do único hospital existente na cidade, de 
natureza filantrópica, conveniada ao SU S, o que gera uma relação de 2,8 leitos/1.000 habitantes. Comparando-se com os hospitais conveniados ao SU S no Brasil, cerca de 6.500, este valor encontra-se de acordo com a maioria das unidades hospitalares, que apresenta cerca de 40 leitos. A distribuição desses leitos concentra-se principalmente em clínica médica $(40,9 \%)$, obstetrícia (29,5\%), clínica cirúrgica $(18,2 \%)$ e pediatria $(11,4 \%)$.

Em 2002, segundo o Sistema de Informações Hospitalares (SIH-SUS), foi registrado um total de 1.085 internações entre a população de João Neiva, ou seja, 6,8\% dos moradores da cidade estiveram internados. Deste total, 832 $(76,7 \%)$ ocorreram no único hospital local. As demais internações ocorreram em hospitais de outros municípios. Dentre as especialidades médicas, a clínica médica registrou a maioria das internações, 566 (52,2\%) e a maior participação dos valores recebidos entre as AIH 's pagas (51,4\%). O valor médio da AIH foi de $\mathrm{R} \$ 233,53$, valor relativamente baixo quando comparado aos hospitais do País, o que demonstra a realização de procedimentos de baixa complexidade, mesmo na atenção hospitalar. 0 tempo médio de permanência nessa especialidade foi de aproximadamente cinco dias, tendo ocorrido 18 óbitos, ou seja, a taxa de mortalidade hospitalar observada em clínica médica foi de 3,2\%. Quando analisamos a relação das internações por sexo, destacam-se as mulheres, devido à obstetrícia; porém ao excluirmos esta especialidade, o número de internações se torna semelhante. A principal causa de internação das mulheres em idade fértil é a gravidez. Entre 1996 e 2000, o número de nascidos vivos oscilou entre 250 a 300. 0 índice de prematuridade atingiu em 2000, por exemplo, 6,3\% dos bebês e aqueles que nasceram com baixo peso, 7,4\%. A gravidez em adolescentes é um problema do País que também existe em João N eiva: em média, cerca de $20 \%$ das adolescentes em idade fértil ficaram grávidas entre 1996 e 2000. Também a proporção de cesarianas é elevada. A Organização Mundial de Saúde (OMS) preconiza que o número de cesarianas seja equivalente a $25 \%$ do total de partos. Ao analisarmos esse dado, veremos que o índice de cesariana está acima do que se preconiza. Um estudo detalhado se faz necessário, para saber o motivo deste elevado índice de cesarianas em João N eiva, entre $40 \%$ e $50 \%$ ao longo de 1996 a 2000.

Uma outra possibilidade para análise das internações é o grupo de causas segundo o CID-
10 por faixa etária. Nesse caso, entre a população idosa, 67\% das internações compreendem quatro grupos do CID-10: 1) doenças do apareIho circulatório $(29,7 \%)$; 2 ) doenças do apareIho respiratório $(19,4 \%) ; 3)$ doenças infecciosas e parasitárias (9,7\%); e 4) doenças do aparelho genito-urinário (8,2\%). O s óbitos também seguem uma relação próxima com aquela observada pelo número de internações, isto é, em 2000, as doenças do aparel ho circulatório e as neoplasias foram as principais responsáveis pelos óbitos entre os idosos no município. Segundo dados do SIOPS (M inistério da Saúde, 2003a), o município ainda não atingiu o percentual mínimo de $15 \%$ de seus recursos próprios aplicados na área da saúde, conforme recomendação da Emenda Constitucional no 29/2000. Esperase que em 2003 esse percentual seja cumprido, pois o município, caminha para atingir tal percentual, se a tendência observada entre 2001 $(12,8 \%)$ e 2002 (14,0\%) for mantida. A Emenda Constitucional estabelece que as esferas que destinavam para o financiamento da saúde, em 2000 , percentuais inferiores a $12 \%$ no caso das unidades da federação, e 15\% no caso dos municípios, deveriam elevá-los gradualmente até 0 oo ano (2005) após a sua aprovação, reduzindo-se essa diferença à razão de, pelo menos, 1/5 por ano (Favaret et al., 2001).

Em relação à receita de impostos e transferências constitucionais legais, corrigindo-se os valores de 2001 pela inflação acumulada desse período para sua comparação com o ano de 2002, demonstra-se que o município perdeu cerca de 0,13\% de sua arrecadação, ou seja, manteve-se estagnado. Já em relação à despesa total com saúde por habitante, observa-se que o município obteve um aumento de 10,3\%, passando de $\mathrm{R} \$ 59,17$ para $\mathrm{R} \$ 65,24$. As transferências federais do SUS, por sua vez, caíram 3,9\%, indo de $\mathrm{R} \$ 83,30$ para $\mathrm{R} \$ 80,04$. A proporção de despesa com investimento em relação à despesa total sofreu uma queda vertiginosa, de $74,3 \%$, passando de 3,5\% para apenas 0,9\%. No total, a despesa com saúde por habitante praticamente se manteve constante entre $2001(\mathrm{R} \$ 142,47)$ e 2002 (R\$145,28) (M inistério da Saúde, 2003b).

\section{Perfil dos idosos entrevistados}

Cada idoso entrevistado foi informado que seria determinado o seu grau de fragilização (com a estratificação de risco - 0, 1, 2 ou 3), a partir de uma série de perguntas. De acordo com 0 
risco encontrado, posteriormente ele seria informado dos resultados da pesquisa e encaminhado para atendimento médico conforme diagrama de fluxo de atendimento apresentado por Veras (2003). A tabela 3 resume os principais resultados da pesquisa. Foram entrevistados 103 idosos. Em um ambulatório da rede pública (unidade de saúde), localizada na área urbana, no centro de João Neiva, entrevistaram-se 59 idosos e na área rural em uma unidade de saúde da família, no Distrito de Acioli, 44 idosos. A maioria era formada por mulheres, isto é, $57,3 \%$ eram do sexo feminino, e $42,7 \%$ do sexo masculino. Os idosos ganham, em média, um salário mínimo, o que está de acordo com os dados do Censo Demográfico do IBGE para 0 ano de 2000, analisados anteriormente para 0 município. 0 apoio social, definido como a pre sença de um familiar que resida com o idoso foi mencionado por $86,4 \%$, isto é, o acol himento dos idosos do município é feito, muitas vezes, pelos próprios parentes. Cerca de $20 \%$ relataram possuir planos privados de saúde, proporção semelhante àqueles com acesso a atendimento odontológico na área urbana. $\mathrm{Na}$ área rural, 0 acesso à odontologia foi elevado: cerca de 40\%, o que sugere uma eficiência da Estratégia de Saúde da Família no alcance das pessoas adscritas e na oferta de serviços desse tipo. Quanto aos fatores de risco, cerca de $5,8 \%$ consideram que a sua "saúde" é excelente; $5,8 \%$, muito boa; $35 \%$, boa; $36,9 \%$, média; e $16,5 \%$, ruim. Em relação à idade, encontrou-se a seguinte distribuição: $29,1 \%$ com 60 a 64 anos; $44,7 \%$ com 65 a 74 anos; $16,5 \%$ com 75 a 79 anos; 4,9\% com 80 a 84 anos; e 4,9\% com 85 anos ou mais. 0 percentual de idosos que foram entrevistados na unidade de saúde da área urbana com consultas agendadas foi de $61 \%$ e na rural $78 \%$. As consultas não agendadas representaram $39 \%$ e $22 \%$ respectivamente. 0 número de consultas médicas nos últimos $12 \mathrm{me}-$ ses anteriores à entrevista teve a seguinte distribuição: 5,8\% não tiveram nenhuma consulta; $10,7 \%$ foram consultados apenas uma vez; $35 \%$, duas ou três vezes; $20,4 \%$, de quatro a seis vezes, e $28,2 \%$, mais de seis vezes. Q uanto ao fator de risco "internação nos últimos 12 meses", 3,9\% reportaram mais detrês vezes; $6,8 \%$, duas ou três vezes; $15,5 \%$, uma vez; $73,8 \%$, nenhuma vez. 0 aumento no número de consultas não seapresenta associado ao número de internações, demonstrando a eficiência das equipes de saúde da família em promover a desospitalização no município de João N eiva. Nos últimos 12 meses, apenas $21,4 \%$ dos idosos estiveram internados pelo menos uma vez pelo fator diabetes mellitus, enquanto nas doenças cardiovasculares foi encontrado um percentual próximo de $20 \%$.

\section{0 risco de fragilização}

Os menores riscos (baixo/médio) foram observados na área urbana (25,5\%). Por outro lado, a proporção de idosos que apresentou risco muito al to foi superior na área rural $(54,5 \%)$, quando comparado à área urbana $(42,4 \%)$, representando uma diferença relativa de $8,5 \%$. $\mathrm{Na}$ área rural, a Estratégia de Saúde da Família consegue captar e manter os idosos nas ações programáticas, pois estabelece um vínculo com a comunidade, conhecendo todas as pessoas pelo nome, o que teria facilitado o registro de um menor número de fal tas às consultas agendadas. Este fato nos leva a crer que a amostra realizada tenha refletido essa situação, esperada, de risco superior entre os idosos da área rural. Em relação ao sexo, o risco de fragilização "muito alto" no sexo masculino foi quase duas vezes superior ao feminino. Quanto à idade, o risco mostrou-se levemente maior nos idosos de 75 anos e mais. Aqui o pequeno tamanho da subamostra realizada pode ter influenciado nos resultados dos riscos baixo, médio e médio/alto (Tabela 4).

\section{Discussão}

No Brasil, a característica histórica de jovialidade da população vem se transformando em uma realidade transitória. Poucos se deram conta de que os tempos passaram e, desde os anos 60, a maioria dos idosos (em números absolutos) vive em países do Terceiro Mundo. Esta é a faixa etária, segundo demonstram as projeções estatísticas, que mais cresce na maioria dos países em desenvolvimento. Segundo o Censo Demográfico do IBGE (2000a), o grupo com 60 anos é o que mais cresce proporcionalmente no Brasil, enquanto a população jovem encontra-se em um processo de desaceleração de crescimento, principalmente a partir de 1970, quando 0 índice foi de 18\% (1970-1980), comparado com o crescimento da década anterior de mais de $31 \%$. De 1980 até 2000, 0 grupo de 0 a 14 anos cresce apenas $14 \%$ contra $107 \%$ dos 60 anos ou mais, sendo que a população como um todo cresce $56 \%$. Isso significa que o novo século iniciou-se com a população idosa crescendo pro- 
Tabela 3

A plicação do instrumento de detecção precoce e de previsi bilidade de agravos entre a população de 60 anos ou mais em duas unidades de saúde do Município de João Neiva (ES) - 2003.

\begin{tabular}{|c|c|c|c|c|c|c|}
\hline \multirow[t]{2}{*}{ Características } & \multicolumn{2}{|c|}{$\begin{array}{c}\text { Área urbana } \\
\text { (n1=59) }\end{array}$} & \multicolumn{2}{|c|}{$\begin{array}{l}\text { Área rural } \\
\text { (n2=44) }\end{array}$} & \multicolumn{2}{|c|}{$\begin{array}{c}\text { Total } \\
(n=103)\end{array}$} \\
\hline & n1 & $\%$ & $\mathrm{n} 2$ & $\%$ & $\mathrm{n}$ & $\%$ \\
\hline \multicolumn{7}{|l|}{ Autopercepção de saúde } \\
\hline Excelente & 5 & $8,5 \%$ & 1 & $2,3 \%$ & 6 & $5,8 \%$ \\
\hline Muito boa & 3 & $5,1 \%$ & 3 & $6,8 \%$ & 6 & $5,8 \%$ \\
\hline Boa & 17 & $28,8 \%$ & 19 & $43,2 \%$ & 36 & $35,0 \%$ \\
\hline M édia & 24 & $40,7 \%$ & 14 & $31,8 \%$ & 38 & $36,9 \%$ \\
\hline Ruim & 10 & $16,9 \%$ & 7 & $15,9 \%$ & 17 & $16,5 \%$ \\
\hline \multicolumn{7}{|l|}{ Hospitalizações } \\
\hline Nenhuma vez & 39 & $66,1 \%$ & 37 & $84,1 \%$ & 76 & $73,8 \%$ \\
\hline $1 \mathrm{vez}$ & 11 & $18,6 \%$ & 5 & $11,4 \%$ & 16 & $15,5 \%$ \\
\hline 2 ou 3 vezes & 6 & $10,2 \%$ & 1 & $2,3 \%$ & 7 & $6,8 \%$ \\
\hline mais de 3 vezes & 3 & $5,1 \%$ & 1 & $2,3 \%$ & 4 & $3,9 \%$ \\
\hline \multicolumn{7}{|l|}{ Consultas médicas } \\
\hline Nenhuma vez & 5 & $8,5 \%$ & 1 & $2,3 \%$ & 6 & $5,8 \%$ \\
\hline $1 \mathrm{vez}$ & 4 & $6,8 \%$ & 7 & $15,9 \%$ & 11 & $10,7 \%$ \\
\hline 2 ou 3 vezes & 23 & $39,0 \%$ & 13 & $29,5 \%$ & 36 & $35,0 \%$ \\
\hline 4 a 6 vezes & 11 & $18,6 \%$ & 10 & $22,7 \%$ & 21 & $20,4 \%$ \\
\hline mais de 6 vezes & 16 & $27,1 \%$ & 13 & $29,5 \%$ & 29 & $28,2 \%$ \\
\hline \multicolumn{7}{|l|}{ Diabetes mellitus } \\
\hline Sim & 13 & $22,0 \%$ & 9 & $20,5 \%$ & 22 & $21,4 \%$ \\
\hline \multicolumn{7}{|l|}{ Doença cardiovascular } \\
\hline Sim & 11 & $18,6 \%$ & 8 & $18,2 \%$ & 19 & $18,4 \%$ \\
\hline \multicolumn{7}{|l|}{ Sexo } \\
\hline Masculino & 24 & $40,7 \%$ & 20 & $45,5 \%$ & 44 & $42,7 \%$ \\
\hline Feminino & 35 & $59,3 \%$ & 24 & $54,5 \%$ & 59 & $57,3 \%$ \\
\hline \multicolumn{7}{|l|}{ Apoio social } \\
\hline Sim & 50 & $84,7 \%$ & 39 & $88,6 \%$ & 89 & $86,4 \%$ \\
\hline Não & 9 & $15,3 \%$ & 5 & $11,4 \%$ & 14 & $13,6 \%$ \\
\hline \multicolumn{7}{|l|}{ Idade } \\
\hline 60-64 anos & 15 & $25,4 \%$ & 15 & $34,1 \%$ & 30 & $29,1 \%$ \\
\hline $65-74$ anos & 25 & $42,4 \%$ & 21 & $47,7 \%$ & 46 & $44,7 \%$ \\
\hline 75 e mais & 19 & $32,2 \%$ & 8 & $18,2 \%$ & 27 & $26,3 \%$ \\
\hline \multicolumn{7}{|l|}{ Renda } \\
\hline Sem rendimento & 2 & $3,4 \%$ & 2 & $4,5 \%$ & 4 & $3,9 \%$ \\
\hline Até 1 salário mínimo & 30 & $50,8 \%$ & 32 & $72,7 \%$ & 62 & $60,2 \%$ \\
\hline De 1 a 2 salários mínimos & 10 & $16,9 \%$ & 4 & $9,1 \%$ & 14 & $13,6 \%$ \\
\hline De 2 a 3 salários mínimos & 4 & $6,8 \%$ & 2 & $4,5 \%$ & 6 & $5,8 \%$ \\
\hline De 3 a 4 salários mínimos & 7 & $11,9 \%$ & 3 & $6,8 \%$ & 10 & $9,7 \%$ \\
\hline De 5 salários mínimos ou mais & 6 & $10,2 \%$ & 1 & $2,3 \%$ & 7 & $6,8 \%$ \\
\hline \multicolumn{7}{|c|}{ Plano privado de assistência à saúde } \\
\hline Sim & 12 & $20,3 \%$ & 9 & $20,5 \%$ & 21 & $20,4 \%$ \\
\hline Não & 47 & $79,7 \%$ & 35 & $79,5 \%$ & 82 & $79,6 \%$ \\
\hline \multicolumn{7}{|l|}{ Acesso atendimento odontológico } \\
\hline Sim & 12 & $20,3 \%$ & 17 & $38,6 \%$ & 29 & $28,2 \%$ \\
\hline Não & 47 & $79,7 \%$ & 27 & $61,4 \%$ & 74 & $71,8 \%$ \\
\hline
\end{tabular}

Fonte: Trabalho de campo. 


\begin{tabular}{|c|c|c|c|c|c|}
\hline \multirow[t]{2}{*}{ Características } & \multicolumn{4}{|c|}{ Grau de risco } & \multirow[t]{2}{*}{ Total } \\
\hline & 0-Baixo & 1-M édio & 2-M édio/Alto & 3-M uito alto & \\
\hline \multicolumn{6}{|l|}{ Localização } \\
\hline Urbana & $9(15,3 \%)$ & $6(10,2 \%)$ & $19(32,2 \%)$ & $25(42,4 \%)$ & $59(100,0 \%)$ \\
\hline Rural & $6(13,6 \%)$ & $2(4,5 \%)$ & $12(27,3 \%)$ & $24(54,5 \%)$ & $44(100,0 \%)$ \\
\hline \multicolumn{6}{|l|}{ Sexo } \\
\hline M asculino & $0(0,0 \%)$ & $2(4,6 \%)$ & $12(27,3 \%)$ & $30(68,2 \%)$ & $44(100,0 \%)$ \\
\hline Feminino & $15(25,4 \%)$ & $6(10,8 \%)$ & $19(32,2 \%)$ & $19(32,2 \%)$ & $59(100,0 \%)$ \\
\hline \multicolumn{6}{|l|}{ Faixa etária } \\
\hline 60 a 64 & $5(16,7 \%)$ & $5(16,7 \%)$ & $7(23,3 \%)$ & $13(43,3 \%)$ & $30(100,0 \%)$ \\
\hline 65 a 74 & $8(17,4 \%)$ & $3(6,5 \%)$ & $16(34,8 \%)$ & $19(41,3 \%)$ & $46(100,0 \%)$ \\
\hline 75 e mais & $2(7,4 \%)$ & $0(0,0 \%)$ & $8(29,6 \%)$ & $17(63,0 \%)$ & $27(100,0 \%)$ \\
\hline
\end{tabular}

Fonte: Trabalho de campo.

porcionalmente oito vezes mais que os jovens e quase duas vezes mais que a população total.

Entre 1950 e 2025, a população de idosos no País crescerá 16 vezes contra cinco vezes da população total, o que colocará o País em termos absolutos como a sexta população de idosos do mundo (mais de 32 milhões de pessoas). As projeções estatísticas demonstram que a proporção de idosos no País passará de 7,3\% em 1991 (11 milhões), para quase 15\% em 2025, que é a atual proporção de idosos da maioria dos países europeus (Carvalho \& Garcia, 2003).

Em um país tão grande como o Brasil, onde os problemas sociais, as dificuldades e as doenças são proporcionais ao número de habitantes, o profissional da saúde, capacitado e integrado à comunidade, pode trazer inúmeros benefícios à população. Tais ações vão desde a assistência à gravidez atéa atenção ao crescimento das crianças, tratamento e prevenção de doenças mais freqüentes, quando uma simples palavra de consolo, ânimo e coragem a uma pessoa enferma atua como um prodigioso remédio.

Hoje, muitos municípios já dispõem de equipes de profissionais do Saúde da Família, que desenvolvem atividades de promoção da saúde, preven ção de doenças e também auxiliam na resolutividade dos serviços (capacidade de resolver a grande maioria dos problemas de saúde). Muito importante também é a contribuição dessas equipes para a desospitalização (tratamento efetivo das pessoas na própria unidade de Saúde da Família, sem a necessidade de atendimento hospitalar). Como o idoso é um grupo etário que tem crescido muito nestes últimos anos, observa-se uma enorme carência de profissionais treinados, com formação específica e cursos reconhecidos pelas faculdades. Até mesmo os profissionais especialistas em geriatria existem em menor número. Reconhece-se apenas cerca de 500 médicos especialistas para atender uma população de 15 milhões de brasileiros, havendo, portanto, uma enorme carência de médicos geriatras. 0 que podemos observar freqüentemente no mercado de trabalho são médicos com idade mais avançada, que possuem uma clientela fiel, prestando assistência a uma população composta basicamente por idosos, não por opção ou formação especifica, mas pelo fato de o profissional e seus pacientes terem envelhecido juntos.

A partir dos resultados obtidos na amostra de idosos em João Neiva, é possível identificar quais os pacientes que têm prioridade de atendimento, por se encaixarem em "pacientes de al to risco". Os de risco baixo têm acesso a um planejamento de intervenções, de forma a mantê-los sau dáveis e ativamente engajados no convívio social. Este instrumento mostrou-se eficiente para organizar e ordenar um serviço de saúde, definindo com clareza os idosos em maior risco e, portanto, que necessitam de tratamento e atenção diferenciada e direcionada para a manutenção de suas capacidades funcionais, preservando e reabilitando sua condição de saúde. Evidenciou-se na amostra realizada que 77,7\% apresentaram risco médio e alto, o que demonstra um elevado percentual da população com significativo grau de fragilização. Esses dados confirmam a tendência observada pelo inquérito domiciliar realizado pelo IBGE no ano de 1998, que registrou para o Estado do Espíri- 
to Santo um total de $82 \%$ de idosos com pelo menos uma doença crônica declarada. Deve-se considerar queo município de João Neiva apresenta quase $100 \%$ de cobertura de PSF e que praticamente toda a população encontra-se cadastrada; porém, só estão incluídos em ações programáticas de saúde alguns grupos risco, tais como grupos de hipertensos e diabéticos. 0 restante da população de idosos não está incluída em ações de prevenção e promoção de saúde.

Os resultados obtidos no estudo realizado apontam para a necessidade de criação de um serviço geriátrico de suporte interdisciplinar, promovendo a reabilitação ediminuindo os riscos de hospitalizações (Guerra, 2002). A amostra deste estudo comportou-se de maneira diferente de outros estudos como Boult et al. (1993) e Veras (2003), nos quais uma pequena parcela da população, cerca de $20 \%$, apresentava risco elevado de fragilidade. A justificativa para a grande diferença observada em nosso estudo é que $68 \%$ dos idosos que compareceram à unidade estavam com consulta agendada, o que significa que já haviam procurado o serviço de saúde anteriormente. As unidades onde foram realizadas as entrevistas são unidades de saúde da família, nas quais a demanda espontânea é significativamente pequena e a população idosa que geralmente procura atendimento são hipertensos, diabéticos, vítimas de AVC ou pacientes em tratamento de fisioterapia.

0 instrumento teve, portanto, uma aplicação maior no que se refere à mensuração do risco de fragilização, ao invés de sua utilização como instrumento de triagem.

\section{Considerações finais}

Em virtude dos fatos mencionados e questionados e diante dos dados coletados e analisados, chegou-se a al gumas considerações:

- Houve um aumento significativo da população idosa em todo o mundo, inclusive no Bra- sil, no Espírito Santo e em João Neiva, sugerindo, portanto, um "novo olhar" sobre os modelos assistenciais vigentes.

- As projeções para o período de 2000 a 2025 dão ao brasileiro uma expectativa média de vida em torno de 80 anos de idade.

- 0 processo de envelhecimento populacional é resultado apenas do declínio da fecundidadee não do aumento de idade.

- A longevidade não deve ser considerada a principal conquista do ser humano, mas sim a qualidade de vida, que deve ser garantida com respeito efelicidade.

- O Brasil é um país que envelhece de maneira rápida, graças aos avanços da tecnologia, engenharia genética e biotecnologia.

- É evidente a eficácia do "questionário", instrumento de triagem rápida, que permite classificar os idosos em diferentes grupos de fragilização, possibilitando a hierarquização da atenção. - Através desse modelo de triagem rápida e de avaliação do risco de fragilização, o idoso é capacitado, avaliado e adequadamente referenciado, permitindo uma melhor organização do atendimento à sua saúde.

- Este instrumento, além de identificar o risco do idoso, permite priorizar atendimento dos que mais necessitam de um serviço geriátrico, promovendo a reabilitação ediminuindo os riscos de hospital ização. Futuros trabal hos deverão ser conduzidos, em um "estudo prospectivo", visando testar a eficiência do instrumento, uma vez que o valor preditivo do "risco de fragilização", como também assinala Veras (2003), não éadequadamente identificado com o desenho do estudo aqui apresentado.

- Este "modelo", que se mostrou tão eficaz no reconhecimento do idoso com maior risco, deveria ser olhado com prioridade pelos serviços assistenciais, por ser: 1) um instrumento de fácil utilização, aplicado no momento da chegada do paciente ao posto de saúde; 2) um instrumento com perguntas simples e objetivas, que permitem que qualquer pessoa treinada 0 aplique em curto espaço de tempo e em diferentes locais. 


\section{Agradecimentos}

Os autores gostariam de agradecer à Coordenação do Curso de Saúde da Família no Espírito Santo, pela possibilidade de realização deste estudo, durante o curso propriamente dito. Em particular, à Secretaria Estadual de Saúde do Espírito Santo eà Escola Nacional de Saúde Pública/Fiocruz.

\section{Colaboradores}

LSA Negri foi responsável pela análise dos dados, elaboração e aplicação do instrumento de coleta de dados na área urbana. GF Ruy, além de ter contribuído para a revisão da literatura, aplicou e apurou o instrumento de coleta de dados na área rural. JB Collodetti contribuiu no levantamento de dados, aplicou o instrumento de coleta de dados na área urbana. LF Pinto, pela análise de dados estatística, levantamento dos dados secundários e revisão da versão final do texto. DR Soranz revisou toda a literatura e participou da análise da consolidação dos resultados.

\section{Referências bibliográficas}

Barros Silva et al. Pesquisa de Avaliação da Descentralização dos Recursos da Atenção Básica: PAB, PACS, PSF, $A F B, V S, I C C N$. Relatório Final. Brasília: Secretaria Executiva do M inistério da Saúde, 2001. Disponível em <http: www.saude.gov.br/bvs>. Acessado em 15 de março de 2002.

Boult $C$ et al. 1993. Screening elders for risk of hospital admission. Journal of the American Geriatric Society 41:811-817.

Brasil 2003. Regulamentação da lei 8.842 que dispõe sobre a Política Nacional do Idoso e dá outras providências. Senado Federal, Braślia.

Carvalho J \& Garcia RA 2003. 0 envelhecimento da população brasileira: um enfoque demográfico. Cadernos de Saúde Pública 19(3):725-733.

Favaret ACSC et al. 2001. Estimativas de impacto da vinculação constitucional de recursos para a Saúde (Emenda Constitucional no 29/2000). Cadernos de Economia da Saúde - Série J. Cadernos no 4. Secretaria de Gestão de Investimentos em Saúde, Brasília.

Guerra HL 2002. Internações hospitalares entre idosos: 0 episódio da Clínica Santa Genoveva e fatores Associados à ocorrência de internações em Bambuí, M inas Gerais. Tese de doutorado. Escola Nacional de Saúde Pública, Fundação Oswaldo Cruz, Rio de Janeiro.

Instituto Brasileiro de Geografia e Estatística (IBGE) 1982. Anuário Estatístico do Brasil. Rio de Janeiro.

Instituto Brasileiro de Geografia e Estatística (IBGE) 2000a. Censo Demográfico.

Instituto Brasileiro de Geografia e Estatística (IBGE) 2000b. Pesquisa Nacional por Amostra de Domicílio (PNAD1998). Suplemento Especial. Acesso e Utilização de Serviços de Saúde. Rio de Janeiro.

Instituto Brasileiro de Geografia e Estatística (IBGE) 2003. Estimativas populacionais. Rio de Janeiro.

Mendes W 2001. Home Care: uma modalidade de audiência de assistência à saúde. Universidade Aberta da Terceira Idade, Rio de Janeiro.

Ministério da Saúde 2002. Caderno de Atenção Básica. Se cretaria de Políticas de Saúde. Departamento de Atenção Básica, Braślila.

M inistério da Saúde 2003a. M anual de Preenchimento do SIOPS para os M unicípios. Secretaria Executiva/Datasus/Coordenação Geral de Informação em Saúde, Brasilia.
Ministério da Saúde 2003b. Cadernos de Informações de Saúde. Secretaria Executiva, Datasus, Brasília. Disponível em <http://www.datasus.gov.br>. Acessado em 17 de novembro de 2003.

Ramos LR 1993. A explosão demográfica da terceira idade no Brasil: uma questão de saúde pública. Gerontologia 1:3-8.

Rede Interagencial de Informações para a Saúde (RIPSA) 2002. Indicadores básicos de saúde no Brasil: conceitos e aplicações. Organização Pan-Americana de Saúde, Brasília.

Reuben DB, Shekelle PG \& Wenger NS. Quality of care for older persons at the dawn of the third millennium. Journal of American Geriatrics Society 51(7, suppl).

Secretaria Estadual de Saúde do Espírito Santo (SES-ES) 2003. Plano Diretor de Regionalização. Espírito Santo.

Silvestre JA \& Costa N eto M M 2003. Abordagem do idoso em programas de saúde da família. Cadernos de Saúde Pública 19(3):839-847.

Tobar F \& Yalour M R 2001. Como fazer teses em saúde pública: conselhos e idéias para formular projetos e redigir teses e informes de pesquisa. (1a ed.). Tradução de M aria Ângela Cançado. Fiocruz, Rio de Janeiro.

Veras RP 2001. Modelos contemporâneos no cuidado à saúde: novos desafios em decorrência da mudança do perfil epidemiológico da população brasileira. Revista USP 51:72-85.

Veras RP, Lourenço R, Martins CSF, Sanchez MA \& Chaves PH 2002. Novos paradigmas do modelo assistencial no setor saúde: conseqüência da explosão populacional dos idosos no Brasil. Medicina Sócia, pp. 11-79. In R Veras (org.). Terceira idade: gestão contemporânea em saúde.Universidade Aberta da Terceira Idade-Universidade do Estado do Rio de Janeiro - Relume Dumará, Rio de Janeiro.

Veras R 2003. Em busca de uma assistência adequada à saúde do idoso: revisão da literatura e aplicação de um instrumento de detecção precoce e de previsibilidade de agravos. Cadernos de Saúde Pública 19(3) 705-715.

Artigo apresentado em 27/12/2003

Aprovado em 27/1/2004

Versão final apresentada em 27/2/2004 